\title{
Effect of Adenine on Clozapine-induced Neutropenia in Patients with Schizophrenia: A Preliminary Study
}

\author{
Ippei Takeuchi ${ }^{1}, 2$, Taro Kishi', Manako Hanya ${ }^{2}$, Junji Uno', Kiyoshi Fujita', Hiroyuki Kamei ${ }^{2}$ \\ 'Department of Psychiatry, Okehazama Hospital, Toyoake, Aichi, ${ }^{2}$ Office of Clinical Pharmacy Practice and Health Care Management, \\ Faculty of Pharmacy, Meijo University, Nagoya, Japan
}

\begin{abstract}
Objective: This study examined the utility of adenine for preventing clozapine-induced neutropenia
Methods: This retrospective study examined the effect of adenine on clozapine-induced neutropenia in patients with treatment-resistant schizophrenia and was conducted at Okehazama Hospital in Japan from July 2010 to June 2013. Adenine was available for use from June 2011 onwards. Twenty-one patients started receiving clozapine treatment from July 2010 to April 2011 (the pre-adenine adoption group), and 47 patients started receiving it from May 2011 to June 2013 (the post-adenine adoption group). The effects of adenine were assessed based on changes in the patients' leukocyte counts and the frequency of treatment discontinuation due to clozapine-induced neutropenia.

Results: Sixty-eight patients were treated with clozapine from July 2010 to June 2013. Of the 21 patients in the pre-adenine adoption group, 4 discontinued treatment due to clozapine-induced neutropenia, whereas only 2 of the 47 patients in the post-adenine adoption group discontinued treatment. The frequency of treatment discontinuation due to clozapine-induced neutropenia was significantly lower in post-adenine adoption group than in the pre-adenine adoption group ( $p=0.047)$.

Conclusion: Adenine decreased the frequency of treatment discontinuation due to clozapine-induced neutropenia. Our data suggest that combined treatment with clozapine and adenine is a safe and effective strategy against treatment-resistant schizophrenia.
\end{abstract}

KEY WORDS: Clozapine; Adenine; Neutropenia; Schizophrenia; Side-effect.

\section{INTRODUCTION}

Clozapine is an effective treatment for refractory schizophrenia. ${ }^{1)}$ Although it has a favorable adverse effects profile, $1 \%$ to $2 \%$ of patients who receive clozapine discontinue treatment due to agranulocytosis. ${ }^{2,3)}$ In addition, clozapine can also cause other hematological side effects including leukopenia, neutropenia, and eosinophilia. ${ }^{4-7)}$

Thus, the full benefit of clozapine can only be achieved if its side effects can be controlled. In Japan, physicians who administer clozapine must conform to the rules set out by the Clozaril Patient Monitoring Service (CPMS).

Accordingly, all patients that receive clozapine are registered with the CPMS and are strictly monitored for reductions in their white blood cell counts every week or

\footnotetext{
Received: November 22, 2014 / Revised: January 13, 2015

Accepted: January 14, 2015

Address for correspondence: Ippei Takeuchi, M.Sharm Department of Psychiatry, Okehazama Hospital, Minamiyakata 3-879, Sakae-cho, Toyoake, Aichi 470-1168, Japan

Tel: +81-562-97-1361, Fax: +81-562-97-8004

E-mail: 134331503@ccalumni.meijo-u.ac.jp
}

two weeks.

Agranulocytosis is defined as a granulocyte count of $<500 / \mathrm{mm}^{3}$, neutropenia is defined as a granulocyte count of $<500-1,500 / \mathrm{mm}^{3}$, and leukopenia is defined as a white blood cell count of $<3,500 / \mathrm{mm}^{3}$. In addition, the CPMS have established strict leukocyte and neutrophil count standards for patients receiving clozapine; i.e., clozapine treatment must be discontinued when a leukocyte count of $<3,000 / \mathrm{mm}^{3}$ or a neutrophil count of $<1,500 / \mathrm{mm}^{3}$ is detected.

Patients with low leukocyte and neutrophil counts are at risk of agranulocytosis and neutropenia, respectively. It is also known that the risk of agranulocytosis or neutropenia is highest in the first 8-12 weeks of treatment. ${ }^{8,9)}$ However, if patients are able to continue receiving clozapine, the risk of agranulocytosis or neutropenia falls over time. For example, a Korean study found that the risk of agranulocytosis or neutropenia gradually reduced during the first 6 years of clozapine treatment and had completely disappeared after 7 years, and a Finnish study reported that $83.9 \%$ of all agranulocytosis cases occurred within the

(c) This is an Open-Access article distributed under the terms of the Creative Commons Attribution Non-Commercial License (http://creativecommons.org/licenses/by-nc/4.0) which permits unrestricted non-commercial use, distribution, and reproduction in any medium, provided the original work is properly cited. 
first 12 months of clozapine treatment. ${ }^{9,10)}$

Lithium has been suggested as a treatment for clozapine-induced neutropenia. ${ }^{11-13)}$ However, lithium is associated with a number of side effects and potentially serious toxicities. ${ }^{14)}$ Other studies have found that treatment with granulocyte-colony stimulating factor (G-CSF) can prevent clozapine therapy being discontinued, ${ }^{15,16)}$ but G-CSF is expensive. ${ }^{17)}$

Therefore, treatments for clozapine-induced neutropenia that have few side effects and are inexpensive are required. Adenine has been reported to be effective against leukocytosis. ${ }^{18,19)}$ However, the effect of adenine on clozapine-induced neutropenia has not been examined. This study was designed to evaluate the efficacy and safety of using adenine in combination with clozapine as a treatment for agranulocytosis and neutropenia.

\section{METHODS}

\section{Subjects and Laboratory Data Sources}

This study examined the cases of 71 patients who received clozapine treatment at Okehazama Hospital from July 2010 to June 2013. The patients' medical charts were reviewed to collect data about the daily dose of clozapine; the duration of clozapine treatment; the daily dose of adenine (10-60 mg/day); the duration of adenine treatment; and the patients' gender, age, leukocyte and neutrophil counts, kidney function (creatinine and blood urea nitrogen levels), and uric acid levels. Adenine was provide by Ohara Pharmaceutical Co. Ltd. (Tokyo, Japan). Complete information was available for 68 patients. We started to use adenine at Okehazama Hospital in June 2011. This study was approved by the ethics committee at Okehazama Hospital. We obtained written informed consent from all patients.

\section{Statistical Analysis}

To test the association between the efficacy and safety of the use of adenine, we firstly tested the relationship between the frequency of clozapine treatment discontinuation and demographic parameters using the chi-square test and Student's $t$-test. Secondly, we used repeated ANOVA and the Mann-Whitney test to compare patients receiving the combination of clozapine and adenine and those administered clozapine alone. Thirdly, we studied associations among the baseline and 24-week kidney function (creatinine and blood urea nitrogen levels) and uric acid levels. $p$-values of $<0.05$ were considered significant. All statistical analyses were performed using the IBM SPSS
Statistics software package Windows (version 22; IBM Co., Armonk, NY, USA).

\section{RESULTS}

Of the 68 patients that were treated with clozapine between July 2010 and June 2013, 54.4\% were male and $45.6 \%$ were female (mean age, 47.5 years). The mean clozapine dose was $425.7 \mathrm{mg}$, and the mean duration of clozapine treatment was 607.4 days (Fig. 1, Table 1).

\section{Treatment Discontinuation due to Neutropenia}

Clozapine treatment was initiated from July 2010 to May 2011 in the pre-adenine adoption group and from June 2011 to June 2013 in the post-adenine adoption group. In the pre-adenine adoption group, the mean age of the patients was 52.9 years, and $61.9 \%$ were male, whereas in the post-adenine adoption group the mean age of the patients was 45.1 years, and $51.1 \%$ were male (Table 2 ). In the post-adenine adoption group, $4.3 \%$ of the patients discontinued clozapine therapy due to clozapine-induced neutropenia (vs. $19 \%$ of the patients in the pre-adenine adoption group; $p=0.047$ ).

\section{Effects of Adenine}

A total of $69.1 \%$ of the patients (47 of 68 ) started receiving clozapine in June 2011 or later. Of these, $25.5 \%$ (12 of 47; group A) were given a combination of clozapine and adenine with the aim of preventing agranulocytosis and neutropenia, $36.2 \%$ (17 of 47 ; group B) were treated with clozapine alone, and $38.3 \%$ (18 of 47 ; group C) were administered adenine after the initiation of clozapine treatment. The latter group was excluded from the analysis. Table 3 show the patients' demographics and treatment characteristics.

Repeated ANOVA showed a non-significant difference between leukocyte counts of groups A and B ( $p=0.052)$. However, the baseline leukocyte count of group B was significantly higher than that of group A (Mann-Whitney test, $p=0.000)$, but no differences in the leukocyte counts of these two groups were detected in other weeks (1 week: $p=0.128,2$ weeks: $p=0.140,3$ weeks: $p=0.404,4$ weeks: $p=0.122$, 24 weeks: $p=0.139$ ). In addition, repeated ANOVA showed a non-significant difference between neutrophil counts of groups $\mathrm{A}$ and $\mathrm{B}(p=0.278)$. However, the baseline neutrophil count of group B was significantly higher than that of group A (Mann-Whitney test, $p=0.000$ ), but no differences in the neutrophil counts of these two groups were detected in other weeks ( 1 week: $p=0.117,2$ 


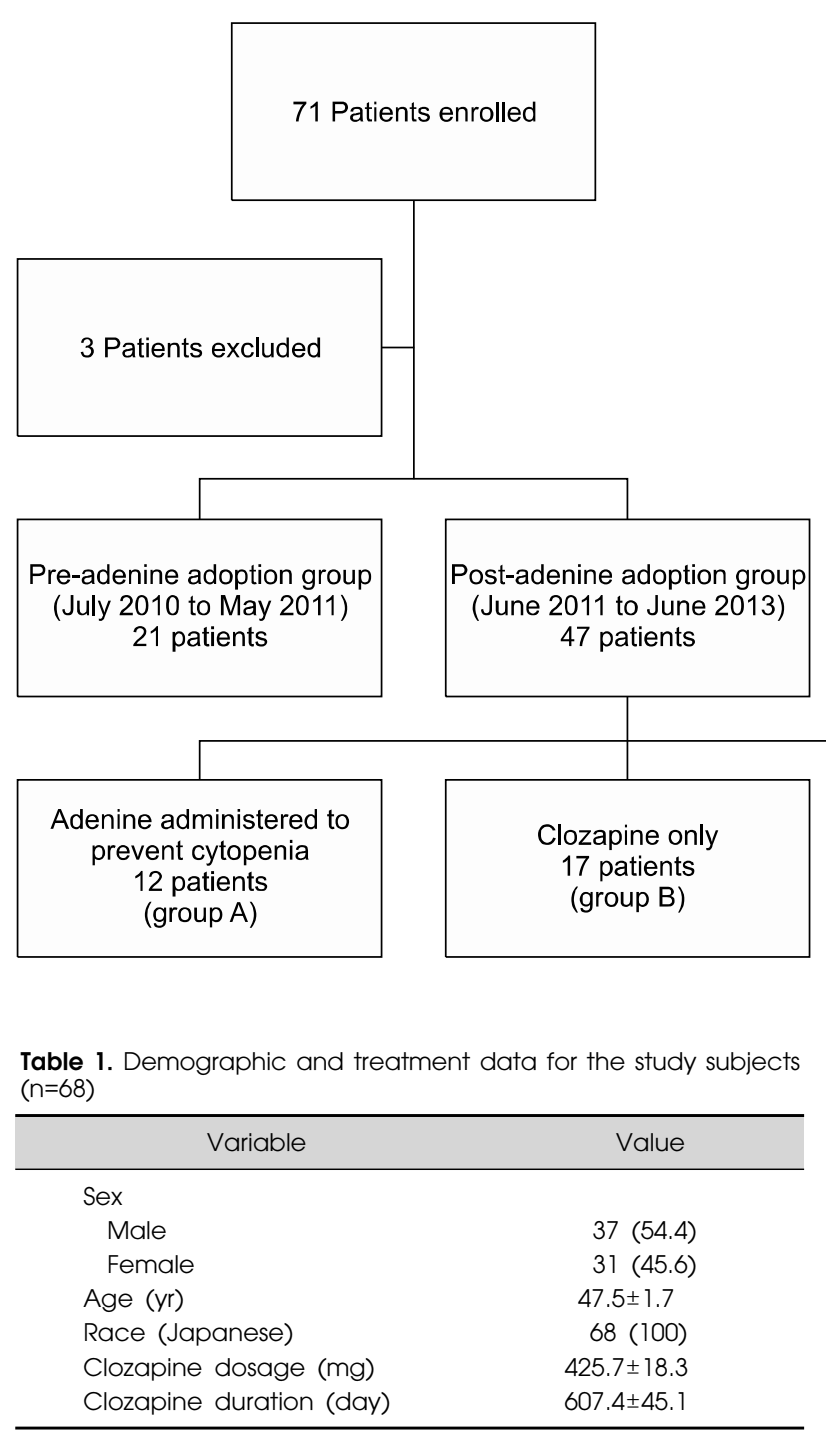

Values are presented as number (\%) or mean \pm standard deviation.

weeks: $p=0.180,3$ weeks: $p=0.890,4$ weeks: $p=0.430,24$ weeks: $p=0.867$ ) (Figs. 2, 3).

\section{Safety of Adenine}

Thirty-five patients received adenine treatment between June 2011 and June 2013 (Fig. 4). One patient exhibited significantly increased uric acid levels at 24 weeks. However, the kidney function (creatinine and blood urea nitrogen levels) and uric acid levels of the 12 patients in group A did not change significantly between the baseline and 24 weeks ( $p=0.949,0.854$, and 0.368 , respectively).

\section{DISCUSSION}

Clozapine was first introduced as a treatment for schizophrenia in Austria in 1969, but it stopped being used in
Adenine administered after the initiation of clozapine treatment 18 patients (group C)

Fig. 1. Flow diagram of the study.

1975 after a report from Finland found that it caused agranulocytosis. However, in 1988 further studies demonstrated that clozapine is effective against refractory schizophrenia.

As a result, clozapine was approved for use as a treatment for refractory schizophrenia in 1989 after the establishment of the CPMS by the United States Food and Drug Administration. The CPMS developed strict leukocyte and neutrophil count standards for patients receiving clozapine, which resulted in reductions in the frequencies of agranulocytosis and mortality. In Japan, adenine is considered to be effective against neutropenia. Regarding the pharmacological function of adenine it is considered to be taken up by DNA of bone-marrow cells and RNA, and then used in nucleic acid biosynthesis ${ }^{20)}$. However, there is no evidence regarding its effectiveness and safety when combined with clozapine. Therefore, we analyzed the effectiveness and safety of adenine in this setting.

We evaluated the efficacy of adenine based on the frequency of clozapine treatment discontinuation due to neutropenia and the changes in the patients' leukocyte and neutrophil counts during treatment. The study subjects were separated into two groups (the pre-adenine adoption and post-adenine adoption groups) according to the date on which their clozapine treatment was initiated. The clozapine treatment discontinuation rate was higher in the pre-adenine adoption group than in the post-adenine 
Table 2. Frequency of clozapine treatment discontinuation in each group $(n=68)$

\begin{tabular}{lccc}
\hline \multicolumn{1}{c}{ Variable } & Pre-adenine adoption group ( $n=21)$ & Post-adenine adoption group ( $n=47)$ & $p$-value* \\
\hline Sex & & & 0.415 \\
Male & $13(61.9)$ & $24(51.1)$ & \\
Female & $8(38.1)$ & $23(48.9)$ & $0.93 .1 \pm 1.9$ \\
Age (yr) & $52.9 \pm 3.4$ & $426.2 \pm 20.5$ & 0.944 \\
Clozapine dosage (mg) & $423.8 \pm 38.2$ & $531.2 \pm 39.8$ & 0.042 \\
Clozapine duration (day) & $777.9 \pm 108.4$ & $2(4.3)$ & 0.047 \\
Discontinuation due to neutropenia & $4(19)$ & $0(0.0)$ & \\
Agranulocytosis & $1(4.8)$ & $2(4.3)$ & \\
Neutropenia & $3(14.3)$ &
\end{tabular}

Values are presented as number (\%) or mean \pm standard deviation.

*By Student's t-test or chi-square test.

Table 3. Post-adenine adoption group $(n=47)$

\begin{tabular}{|c|c|c|c|}
\hline Variable & Group $A(n=12)$ & Group B $(n=17)$ & Group C $(n=18)$ \\
\hline \multicolumn{4}{|l|}{ Sex } \\
\hline Male & $5(41.7)$ & $11(64.7)$ & $8(44.4)$ \\
\hline Female & $7(58.3)$ & $6(35.3)$ & $10(55.6)$ \\
\hline Age $(y r)$ & $50.3 \pm 3.9$ & $41.8 \pm 3.5$ & $44.8 \pm 2.4$ \\
\hline Clozapine dosage (mg) & $423.8 \pm 29.4$ & $417.6 \pm 39.7$ & $404.2 \pm 32.7$ \\
\hline Clozapine duration (day) & $777.9 \pm 59.9$ & $517.2 \pm 65.8$ & $432.3 \pm 64.2$ \\
\hline Adenine dosage (mg) & $36.7 \pm 3.3$ & & $48.3 \pm 3.6$ \\
\hline Discontinuation due to neutropenia & $0(0.0)$ & $0(0.0)$ & $2(0.11)$ \\
\hline Agranulocytosis & $0(0.0)$ & $0(0.0)$ & $0(0.00)$ \\
\hline Neutropenia & $0(0.0)$ & $0(0.0)$ & $2(0.11)$ \\
\hline
\end{tabular}

Values are presented as number (\%) or mean \pm standard deviation.

Adenine was administered to prevent cytopenia in group $A$.

Clozapine alone was administered in group B.

Adenine was administered after the initiation of clozapine in group C.

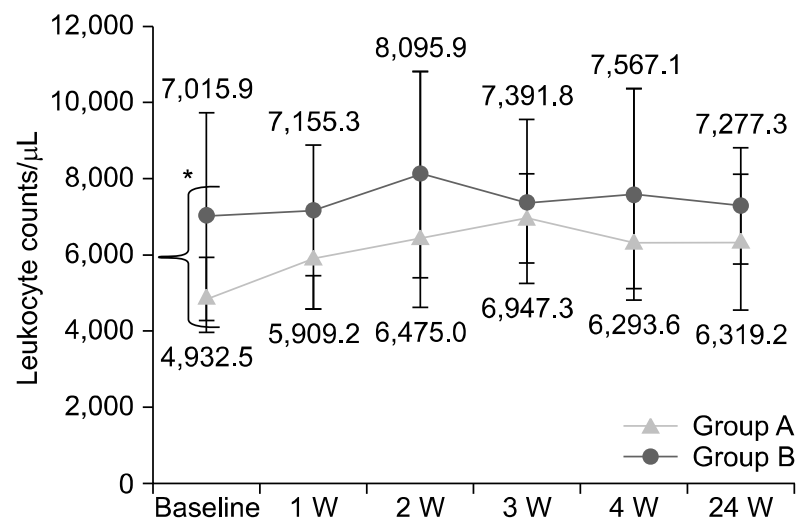

Fig. 2. Changes in the patients' leukocyte counts. $p<0.05$ vs. group B (Mann-Whitney-U test).

Adenine was administered to prevent cytopenia in group $A$; clozapine alone was administered in group B.

adoption group. However, the patients in the pre-adenine adoption group were significantly older than those in the post-adenine adoption group (mean age: $52.9 \pm 3.4$ years vs. $45.1 \pm 1.9$ years; $p=0.033$; Table 2 ), and agranulocytosis is linked with older age. ${ }^{3,10)}$ Thus, our results might have been affected by the age difference between the two

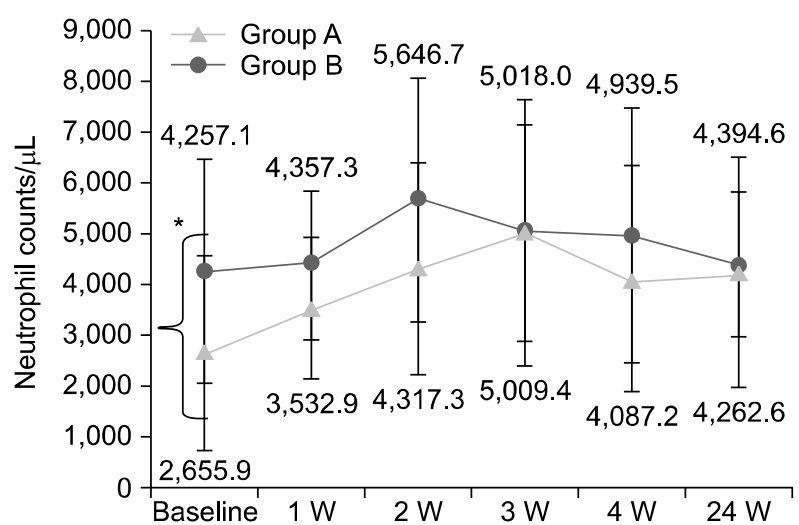

Fig. 3. Changes in the patients' neutrophil counts. $p<0.05$ vs. group B (Mann-Whitney-U test).

Adenine was administered to prevent cytopenia in group $A$ clozapine alone was administered in group B.

groups. On the other hand, the clozapine treatment discontinuation rate of the post-adenine adoption group was significantly better than that of the pre-adenine adoption group despite the former group containing more than double the number of patients than the latter group. This suggested that adenine is effective to reduce the rate of treat- 


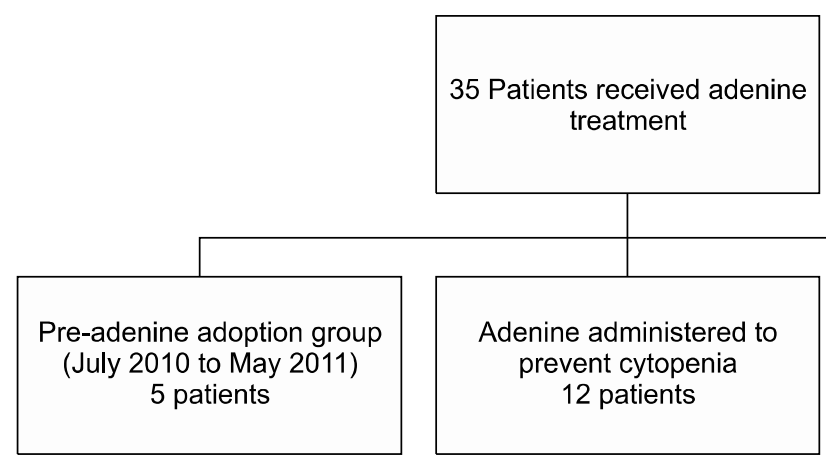

ment discontinuation in schizophrenia patients receiving clozapine.

The baseline leukocyte and neutrophil counts of group A were significantly lower than those of group B (both $p=0.000$ ). However, repeated ANOVA showed a non-significant difference between leukocyte and neutrophil counts of groups A and B ( $p=0.052,0.278$, respectively) and no such differences were detected in other weeks. There were no cases of treatment discontinuation due to neutropenia in groups A or B; however, two cases were seen in group $\mathrm{C}$. However, the mechanism by which adenine prevents agranulocytosis could not be elucidated due to the low number of cases. In addition, the causative mechanism of agranulocytosis differs from that of neutropenia. ${ }^{9)}$ Whilst adenine was demonstrated to be effective at preventing agranulocytosis/neutropenia in group A, no such effect was seen in group $\mathrm{C}$. Therefore, it might be necessary to administer adenine before clozapine treatment. Whilst the finding that the patients' leukocyte and neutrophil counts were markedly reduced by clozapine treatment is an issue, the fact that some patients exhibited low baseline leukocyte and neutrophil counts is not, as leukocyte and neutrophil counts vary between individuals. ${ }^{21)}$ Whilst it has been suggested that transient neutropenia is harmless, ${ }^{5)}$ clozapine treatment is subject to strict regulation by the CPMS, including with regard to the minimum neutrophil count, to ensure patient safety.

At the very least, it can be said that adenine helps to prevent neutropenia when administered before the start of clozapine treatment. This is supported by the low baseline leukocyte and neutrophil counts of the patients in group A, who will not develop agranulocytosis or neutropenia. However, clinicians should take patients' age and sex as well as the dosing period into account when administering adenine in combination with clozapine. Lithium is commonly used to treat clozapine-induced agranulocytosis and neutropenia; however, it is a mood stabilizing drug and can have side effects, including tremors, excessive
Adenine administered after the initiation of clozapine treatment 18 patients

Fig. 4. Flow diagram of the patients that received adenine treatment.

thirst, nausea, diarrhea, epigastric pain, muscle weakness, and fatigue. Therefore, physicians should closely monitor patients who take lithium. In this study, one patient's uric acid levels appeared to be increased by the administration of adenine. However, none of the other patients suffered side effects. The patient with elevated uric acid levels was treated with febuxostat, and their uric acid levels normalized. Overall, our findings suggest that adenine has a high potential as adjunct therapy with clozapine.

This study has several limitations: (1) It is reported that the frequency of clozapine-induced agranulocytosis is about $1 \%$ to $2 \%^{2,3)}$ and that of clozapine-induced neutropenia is about $10.28 \%$; therefore, the small number of patients enrolled in our study is an issue. (2) Our study was a cohort study; however, to examine the effects of adenine in detail a randomized double-blind trial involving adenine and a placebo must be performed. (3) This study did not examine the effects of concomitant medications or different adenine doses.

Among patients with refractory schizophrenia who were treated with clozapine, adenine administration resulted in a significantly lower frequency of treatment discontinuation due to neutropenia as well as increases in the patients' leukocyte and neutrophil counts. Combination treatment with clozapine and adenine will benefit patients who were previously unable to start clozapine treatment due to a low leukocyte or neutrophil count and those who find it difficult to continue with clozapine treatment due to marked reductions in their leukocyte and/or neutrophil counts. We therefore suggest that adenine can be used safely, providing that patients' leukocyte and neutrophil counts are monitored closely.

The efficacy and safety of combination treatment involving clozapine and adenine were demonstrated in this trial. Further data are needed to confirm our findings, with well-designed randomized controlled trials being ideal. 


\section{REFERENCES}

1. Kane J, Honigfeld G, Singer J, Meltzer H. Clozapine for the treatment-resistant schizophrenic. A double-blind comparison with chlorpromazine. Arch Gen Psychiatry 1988;45:789-796.

2. Alvir JM, Lieberman JA, Safferman AZ, Schwimmer JL, Schaaf JA. Clozapine-induced agranulocytosis. Incidence and risk factors in the United States. $N$ Engl $J$ Med 1993;329:162-167.

3. Alvir JM, Lieberman JA. Agranulocytosis: incidence and risk factors. J Clin Psychiatry 1994;55 Suppl B:137-138.

4. Klimke A, Klieser E. The atypical neuroleptic clozapine (Leponex)--current knowledge and recent clinical aspects. Fortschr Neurol Psychiatr 1995;63:173-193.

5. Hummer M, Kurz M, Barnas C, Saria A, Fleischhacker WW. Clozapine-induced transient white blood count disorders. J Clin Psychiatry 1994;55:429-432.

6. Jann MW, Grimsley SR, Gray EC, Chang WH. Pharmacokinetics and pharmacodynamics of clozapine. Clin Pharmacokinet 1993;24:161-176.

7. Latif Z, Malik MA, Jabbar F, Ahmed Y, McDonough C. Clozapine-induced late leukopenia. Ir J Med Sci 2012;181. 139-141.

8. Lieberman JA, Yunis J, Egea E, Canoso RT, Kane JM, Yunis EJ. HLA-B38, DR4, DQw3 and clozapine-induced agranulocytosis in Jewish patients with schizophrenia. Arch Gen Psychiatry 1990;47:945-948.

9. Kang BJ, Cho MJ, Oh JT, Lee Y, Chae BJ, Ko J. Long-term patient monitoring for clozapine-induced agranulocytosis and neutropenia in Korea: when is it safe to discontinue CPMS? Hum Psychopharmacol 2006;21:387-391.

10. Lahdelma L, Appelberg B. Clozapine-induced agranulocytosis in Finland, 1982-2007: long-term monitoring of patients is still warranted. J Clin Psychiatry 2012:73:837-842.

11. Kanaan RA, Kerwin RW. Lithium and clozapine rechallenge: a retrospective case analysis. J Clin Psychiatry 2006;67:
756-760.

12. Palominao A, Kukoyi O, Xiong GL. Leukocytosis after lithium and clozapine combination therapy. Ann Clin Psychiatry 2010;22:205-206.

13. Bender S, Linka T, Wolstein J, Gehendges S, Paulus HJ, Schall U, et al. Safety and efficacy of combined clozapinelithium pharmacotherapy. Int $J$ Neuropsychopharmacol 2004; 7:59-63.

14. Dennison U, Clarkson M, O'Mullane J, Cassidy EM. The incidence and clinical correlates of lithium toxicity: a retrospective review. Ir J Med Sci 2011;180:661-665.

15. Rajagopal G, Graham JG, Haut FF. Prevention of clozapineinduced granulocytopenia/agranulocytosis with granulocytecolony stimulating factor ( $G$-CSF) in an intellectually disabled patient with schizophrenia. $J$ Intellect Disabil Res 2007; 51:82-85.

16. Khan AA, Harvey J, Sengupta S. Continuing clozapine with granulocyte colony-stimulating factor in patients with neutropenia. Ther Adv Psychopharmacol 2013;3:266-271.

17. Timmer-Bonte JN, Adang EM, Termeer E, Severens JL, Tjan-Heijnen VC. Modeling the cost effectiveness of secondary febrile neutropenia prophylaxis during standarddose chemotherapy. J Clin Oncol 2008;26:290-296.

18. Reznikoff P. The treatment of agranulocytosis with adenine sulphate. J Clin Invest 1933;12:45-53.

19. Owan T. The effects of adenine on the leukopenia after x-irradiation. I. Nihon Igaku Hoshasen Gakkai Zasshi 1962;21:1170-1178.

20. Lajtha LG, Oliver R, Ellis F. Incorporation of ${ }^{32} P$ and adenine ${ }^{14} C$ into DNA by human bone marrow cells in vitro. Br J Cancer 1954;8:367-379.

21. Okada Y, Hirota T, Kamatani Y, Takahashi A, Ohmiya H, Kumasaka $\mathrm{N}$, et al. Identification of nine novel loci associated with white blood cell subtypes in a Japanese population. PLoS Genet 2011;7:e1002067. 